\title{
Diabetes Mellitus Management Among Patients with Limited English Proficiency: A Systematic Review and Meta-Analysis
}

\author{
Jane W. Njeru, MB, ChB ${ }^{7}$, Mark L. Wieland, MD, MPH' , Gracia Kwete, MD 2,3 , Eugene M. Tan, MD \\ Carmen Radecki Breitkopf, PhD ${ }^{5}$, Amenah A. Agunwamba, PhD ${ }^{6}$, Larry J. Prokop, MLS , and \\ M. Hassan Murad, MD, MPH $H^{6,8}$
}

\begin{abstract}
'Division of Primary Care Internal Medicine, Mayo Clinic, Rochester, MN, USA; ${ }^{2}$ Mayo Clinic School of Medicine, Mayo Clinic College of Medicine and Science, Rochester, MN, USA; ${ }^{3}$ Massachusetts General Hospital, Boston, MA, USA; ${ }^{4}$ Division of Infectious Diseases, Mayo Clinic, Rochester, MN, USA; ${ }^{5}$ Division of Health Care Policy and Research, Mayo Clinic, Rochester, MN, USA; ${ }^{\circ}$ Robert D. and Patricia E. Kern Center for the Science of Health Care Delivery, Mayo Clinic, Rochester, MN, USA; ${ }^{7}$ Mayo Medical Library, Mayo Clinic, Rochester, MN, USA; ${ }^{8}$ Division of Preventive, Occupational, and Aerospace Medicine, Mayo Clinic, Rochester, MN, USA.
\end{abstract}

BACKGROUND: Patients with limited English proficiency (LEP) and type 2 diabetes mellitus (T2DM) have several health disparities, including suboptimal patient-provider interactions, poorer glycemic control, and T2DM complications. Understanding existing interventions for improving T2DM outcomes in this population is critical for reducing disparities.

METHODS: We performed a systematic review of randomized controlled trials (RCTs) and observational studies examining the effectiveness of interventions in improving T2DM outcomes among patients with LEP in North America. Quality was assessed using the Cochrane risk of bias tool for RCTs and the Newcastle-Ottawa Scale for nonRCT studies. Meta-analysis was conducted using the random-effects model.

RESULTS: Fifty-four studies, 39 of which reported sufficient data for meta-analysis of glycemic control, were included. The interventions were associated with a statistically significant reduction in hemoglobin $\mathrm{A}_{1 \mathrm{c}}\left(\mathrm{HbA}_{1 \mathrm{c}}\right)$ (weighted difference in means, $-0.84 \%$ [95\% CI, -0.97 to $-0.71])$ that was, however, very heterogeneous across studies $\left(\mathrm{I}^{2}=95.9 \%\right)$. Heterogeneity was explained by study design (lower efficacy in RCTs than non-RCTs) and by intervention length and delivery mode (greater reduction in interventions lasting $<6$ months or delivered face-to-face); $P<0.05$ for all three covariates. The interventions were also associated in most studies with improvement in knowledge, self-efficacy in diabetes management, quality of life, blood pressure, and low-density lipoprotein cholesterol.

DISCUSSION: Multiple types of interventions are available for T2DM management in patients with LEP. Multicomponent interventions delivered face-to-face seem most effective for glycemic control. More research is needed to better understand other aspects of multicomponent interventions that are critical for improving important outcomes among patients with T2DM and LEP.

Electronic supplementary material The online version of this article (https://doi.org/10.1007/s11606-017-4237-1) contains supplementary material, which is available to authorized users.

Received March 6, 2017

Revised June 14, 2017

Accepted November 13, 2017

Published online December 18, 2017
KEY WORDS: chronic disease; diabetes; disease management; health communication; language barriers; limited English proficiency.

J Gen Intern Med 33(4):524-32

DOI: $10.1007 / \mathrm{s} 11606-017-4237-1$

(c) Society of General Internal Medicine 2017

\section{INTRODUCTION}

In the United States, type 2 diabetes mellitus (T2DM) disproportionately affects ethnic and racial minority groups, 1, 2 including immigrant and refugee populations. ${ }^{3}$ Compared with nonminority groups, ethnic and racial minority groups with T2DM have worse diabetes-related morbidity. ${ }^{4,5}$ Multiple factors have a role in these disparities, including lower levels of medication adherence, poorer dietary quality, and lower levels of physical activity among some ethnic and racial minority groups. ${ }^{6-8}$ These may be further influenced by social, economic, environmental, and linguistic vulnerability, as well as lower levels of health literacy. $^{9-11}$

A growing subset of the US immigrant and refugee population has limited English proficiency (LEP), defined as a limited ability to read, speak, write, or understand English. ${ }^{12,13}$ The number of people with LEP increased by $80 \%$ in the past two decades; they currently constitute approximately $9 \%$ of the US population. LEP is an important risk factor in health disparities and has been linked to overall poor health status. It is associated with limitations in access to health care, ${ }^{14}$ decreased understanding of medical information, ${ }^{15}$ lower adherence to preventive services, ${ }^{16}$ and suboptimal disease-specific outcomes across multiple conditions. ${ }^{17-19}$ In patients with T2DM, LEP has been associated with longer hospital stays, ${ }^{20}$ suboptimal patient-provider interactions, ${ }^{21}$ and poorer glycemic control. ${ }^{22}$ The appropriate use of medical interpreters or provision of $100 \%$ language-concordant providers only partially mitigates these disparities. $^{23,} 24$

Although patients with LEP have widely heterogeneous characteristics, including country of origin, culture, ethnicity, race, and sociodemographic factors, they often share the common experience of facing linguistic barriers in their interactions with health care teams and systems. This 
barrier can represent a unique challenge for patients living with chronic conditions such as T2DM, in which disease management often requires some understanding of the condition plus ongoing self-management. Because immigrants and refugees represent the largest sector of population growth in the United States, a comprehensive understanding of health care strategies for improving T2DM management among these groups is a critical public health imperative. In addition, because patients with LEP have T2DMrelated health disparities, an understanding of existing strategies is an important step in developing systematic health care changes to reduce these disparities.

The overall purpose of this systematic review and metaanalysis was to describe and summarize interventions in T2DM management and outcomes among patients with LEP in North America and to identify intervention characteristics associated with success.

\section{METHODS}

This systematic review was conducted according to a protocol established a priori and is reported according to the PRISMA statement (Preferred Reporting Items for Systematic Reviews and Meta-Analyses). ${ }^{25}$

\section{Literature Search Strategy}

We developed a search strategy in consultation with an expert reference librarian, who then implemented the electronic literature search. We searched the following databases: Embase 1988 to 2016 week 29; Ovid MEDLINE In-Process and Other Non-Indexed Citations and Ovid MEDLINE 1946 to present; EBM Reviews - Cochrane Central Register of Controlled Trials June 2016; EBM Reviews - Cochrane Database of Systematic Reviews 2005 to July 20, 2016; and Scopus 2005 to July 2016. We used a controlled vocabulary and supplemented it with key words for comparative studies of interventions for T2DM in people with LEP in North America. The detailed search strategy is available in the Appendix.

\section{Eligibility Criteria}

Eligible studies were randomized controlled trials (RCTs) and observational studies that enrolled patients with LEP and T2DM and tested interventions for T2DM (excluding gestational diabetes) management and outcomes. To be included, studies had to fulfill the following criteria: 1) conducted in North America, 2) included patients with LEP, 3) compared the intervention group with a control group or reported measures before and after the intervention, 4) written in English, and 5) reported specific T2DM outcomes.

\section{Data Extraction}

Six investigators working independently and in duplicate screened titles and abstracts to identify eligible studies. Any disagreements resulted in inclusion of the abstract in the next stage of screening. Full texts of all included abstracts were obtained and reviewed in duplicate. Disagreements were resolved by discussion and consensus. The agreement among reviewers was adequate.

Data were abstracted independently and in duplicate using a standardized form that included the following: publication year, design, population studied, percentage of patients with LEP, setting, intervention characteristics, and T2DM-related outcomes.

\section{Data Analysis}

The primary aim of this study was to review interventions for T2DM management among patients with LEP and to identify intervention characteristics associated with improved T2DM outcomes. Therefore, specific study and intervention characteristics were examined and compared across the studies included in the review.

\section{Quality of Studies}

To assess the quality of studies, we used the Cochrane risk-ofbias assessment tool for RCTs and the Newcastle-Ottawa Scale for the assessment of non-RCT studies. ${ }^{26,27}$

\section{Statistical Analysis}

When data were available and appropriate for statistical pooling, a meta-analysis was conducted to analyze the effectiveness of the intervention on glycemic control. The mean (SD) hemoglobin $\mathrm{A}_{1 \mathrm{c}}\left(\mathrm{HbA}_{1 \mathrm{c}}\right)$ levels were extracted from each study and transformed into a weighted mean difference; 95\% confidence intervals (CIs) were calculated and combined using random-effects models. ${ }^{28}$ Heterogeneity was quantified using the $\mathrm{I}^{2}$ statistic; $\geq 50 \%$ was considered evidence of significant heterogeneity. ${ }^{29}$ A subgroup analysis was performed to explore heterogeneity on the basis of several covariates determined a priori: type of study, population, proportion of patients with LEP, duration of study, and type and number of intervention components. All analyses were conducted with Stata 14 software (StataCorp LP, College Station, TX).

\section{RESULTS}

\section{Search Results and Included Studies}

The electronic search yielded 2459 potentially eligible studies. After screening, 54 studies met the inclusion criteria; 39 reported sufficient data for meta-analysis of glycemic control. Figure 1 shows the PRISMA flow diagram.

\section{Methodological Quality of Studies and Risk of Bias}

Seventeen of the 24 RCTs (71\%) included a description of random sequence generation, and 19 (79\%) had complete 


\section{2,459 Records identified through database searching and removal of duplicates}

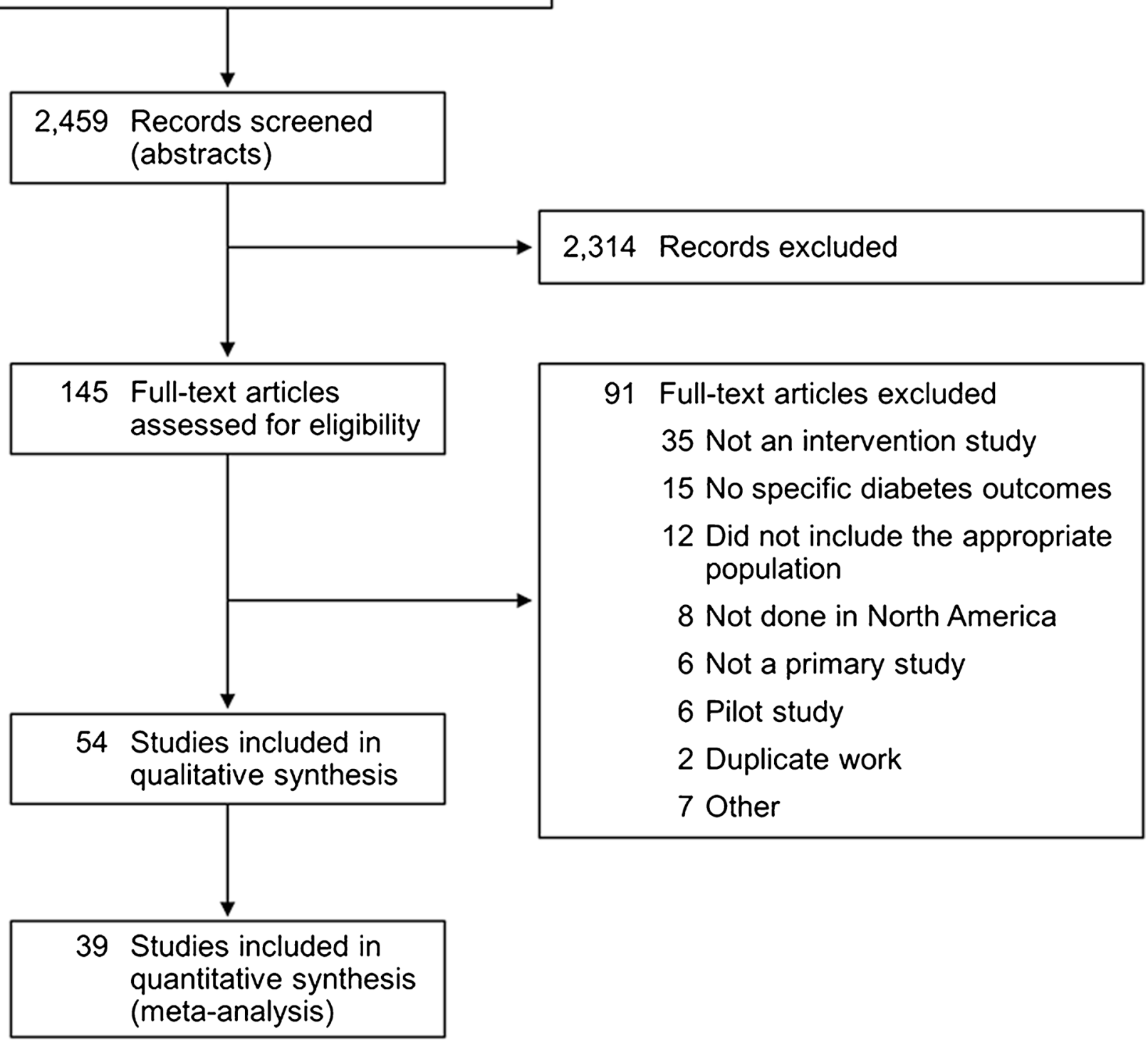

Figure 1 PRISMA flow diagram.

outcome data (Supplemental Table 1). Representativeness of cohorts and cases was good, and ascertainment of exposure and outcomes was excellent (Supplemental Tables 2 and 3). Duration of follow-up was greater than 6 months for most studies. The characteristics of all included studies are summarized below and in the supplemental data (Supplemental Table 4, study design and intervention characteristics; Supplemental Table 5, study outcomes and potential sources of bias). Given the nature of interventions in most of the studies, participant and personnel blinding was not possible. Other possible sources of bias included self-report of some outcomes. Overall, the risk of bias was moderate.

\section{Characteristics of Included Studies}

Populations and Settings. The populations studied were heterogeneous, and the majority of studies (48\%) included
Hispanic patients, with or without other ethnic groups (e.g., African Americans or Asians). Three studies included diverse Asian patients, one study included only Hmong patients, and another enrolled only Marshallese patients (immigrants from Marshall Islands in the Pacific Ocean). When language information was supplied ( $96 \%$ of studies), the language most commonly spoken by patients with LEP was Spanish $(88 \%)$. Other languages included Korean, Cantonese, Mandarin, Tagalog, Vietnamese, Samoan and Tonga, Hmong, and Marshallese. All studies included in this review had patients with LEP. However, the description of patients varied, and characteristics often had to be inferred from tables - in 24 studies (44.4\%), the presence of patients with LEP was only implied and not reported directly. Of the 39 studies included in the meta-analysis, in 19 studies patients with LEP comprised more than $50 \%$ of the participants. 
Of the 54 studies included in this review, 34 (63\%) were performed in community clinics and centers, $11(20 \%)$ in teaching hospitals or tertiary clinics, four $(7 \%)$ in large metro areas, two (4\%) in primary care clinics, and one $(2 \%)$ in an emergency department.

Interventions. The interventions tested varied and included the following: diabetes case management, diabetes selfmanagement support, in-person diabetes education, telephone-based (voice or text) education or reminders, Internet-based education or reminders, and peer-based support groups. In $58.4 \%$ of the studies, the intervention included several of these components in varying combinations. The frequency of intervention delivery depended on the intervention type and ranged from a single 5-min video to weekly or monthly classes lasting several months. Study lengths ranged from a few hours to 5 years; $60.4 \%$ lasted 6 months or longer.

Intervention personnel included case managers, diabetes educators, dietitians, nurses, community health workers (CHWs), peer leaders, and pharmacists. In $55.6 \%$ of the studies, only one type of interventionist was involved (most commonly a CHW), whereas in $38.9 \%$, interprofessional teams delivered the intervention.

Most studies (94\%) reported accommodations for patients with LEP. These included the following, either alone or in some combination: linguistic and/or cultural adaptation of interventions (84\%) and use of interpreters and/or languageconcordant interventionists $(31 \%)$.

Outcomes. Multiple T2DM-related outcomes were examined. For this review, outcomes were divided into five main categories, as described in "Methods." Varying combinations of these outcomes were examined and reported, with most studies including at least three of the five categories.

$\mathrm{HbA}_{1 \mathrm{c}}$ was reported as an outcome in 50 studies (92.6\%), with $38(76 \%)$ reporting statistically significant improvement. In some studies, improvement in $\mathrm{HbA}_{1 \mathrm{c}}$ was limited to a subgroup of patients with poorer glycemic control at baseline.

Several other T2DM-related outcomes were examined and reported (Supplemental Figure 1). Fasting blood glucose was reported in five studies, three of which showed improvement. Of the 18 studies reporting low-density lipoprotein cholesterol (LDL-C) values, 12 showed significant improvement. These studies more often used face-to-face interventions over multiple sessions. Body mass index (BMI) improved in 8 of the 18 studies in which this was reported. Blood pressure was an outcome in 20 studies, 11 of which reported improvement. These studies often lasted longer than 6 months and used interventions that incorporated more than three sessions.

Diabetes self-management was reported as an outcome in 14 of 38 studies. Eight of 13 studies showed improvement in dietary outcomes. These studies lasted longer than 6 months, typically had a single-component intervention, and enrolled fewer than $50 \%$ of patients with LEP. Eleven of 14 studies reported improved physical activity. These studies were generally longer than 6 months and included more than three faceto-face sessions in the intervention.

T2DM-related quality of life was reported as an outcome for only eight studies (14.5\%). Of these, six reported improvement in quality of life; they were generally longer than 6 months and involved multiple face-to-face sessions.

\section{Meta-Analysis of Glycemic Control and Subgroup Analysis}

Thirty-nine studies provided data sufficient for meta-analysis of $\mathrm{HbA}_{1 \mathrm{c}}$, with a total of 10,300 patients enrolled. The results are shown in Figure 2. Many of these studies were not RCTs, had fewer than $50 \%$ of patients with LEP, and included Hispanic populations. These interventions more often had a single component, lasted longer than 6 months, and included more than three face-to-face sessions. When all studies were included in analysis, the interventions were associated with a statistically significant reduction in $\mathrm{HbA}_{1 \mathrm{c}}$ (weighted difference in means, $-0.84 \%$ [95\% CI, -0.97 to -0.71$]$ ); however, the values were very heterogeneous across studies $\left(\mathrm{I}^{2}=95.9 \%\right.$; Fig. 2). The results of a sensitivity analysis of only RCTs with $\geq 50 \%$ of patients having LEP were consistent with the main analysis, showing a change (reduction) in $\mathrm{HbA}_{1 \mathrm{c}}$ of -0.64 (95\% CI, -0.98 to -0.31 ; Fig. 3 ).

Subgroup analysis of all the studies showed that the effect on $\mathrm{HbA}_{1 \mathrm{c}}$ was significantly higher in observational studies versus RCTs and in studies with shorter interventions ( $<6$ months; Table 1). Subgroup analysis of the RCTs showed that the effect on $\mathrm{HbA}_{1 \mathrm{c}}$ was higher in studies with shorter and face-to-face interventions (Table 1).

Although the differences were not statistically significant, other factors associated with greater $\mathrm{HbA}_{1 \mathrm{c}}$ reduction in the subgroup analyses were as follows: fewer than $50 \%$ of patients with LEP, multicomponent interventions, inclusion of more than three sessions, and having a CHW as the interventionist. Outcomes Other Than Glycemic Control. Other patientreported outcomes, including attitude toward diabetes, diabetes knowledge, and self-efficacy in diabetes management, were also reported in varying combinations in 18 of the 54 studies. Data for these outcomes were heterogeneous or insufficient for meta-analysis. However, significant improvements in these outcomes were reported by most studies (range, 60$83 \%$ ), particularly those studies with interventions that were face-to-face, multicomponent, and included multiple sessions.

\section{Quality of Evidence}

The quality of evidence ${ }^{30}$ (i.e., certainty in the results) was moderate for the effectiveness of various multicomponent interventions on $\mathrm{HbA}_{1 \mathrm{c}}$. The reason for this rating is that the evidence was derived primarily from RCTs that had some methodological limitations. Heterogeneity was partially explained by subgroup analyses. Publication bias could not be 


\section{RCT}

Anderson, 2010

Ayala, 2015

Brown, 2002

Castejón, 2013

Chamany, 2015

Corkery, 1997

Glasgow, 2012

Heisler, 2014

Kim, 2009

Lujan, 2007

Palmas, 2014

Prezio, 2013

Schillinger, 2009

Shea, 2007

Toobert, 2011

Welch, 2011

Subtotal $1^{2}=98.0 \%(P<.001)$

\section{Observational study}

Barrera, 2014

Brown, 2007

Clingerman, 2008

Culhane-Pera, 2005

Culica, 2008

Davidson, 2003

de Peralta, 2005

Duggan, 2014

Gilmer, 2005

$\mathrm{Hu}, 2014$

Joshu, 2007

Kane, 2015

Kenya, 2014

Leal, 2008

Metghalchi, 2008

Ryabov, 2010

Salto, 2011

Spencer, 2011

Tomioka, 2014

Tsui, 2013

Vincent, 2007

Weltin, 2012

Wheeler, 2012

Subtotal $1^{2}=94.6 \%(P<.001)$

Overall $\mathrm{I}^{2}=95.9 \%(P<.001)$

Note: Weights are from random-effects analysis
52

90

80

55

74

52

97

55

37

16

50

56

90

40

90

41

67

66

66

65

90
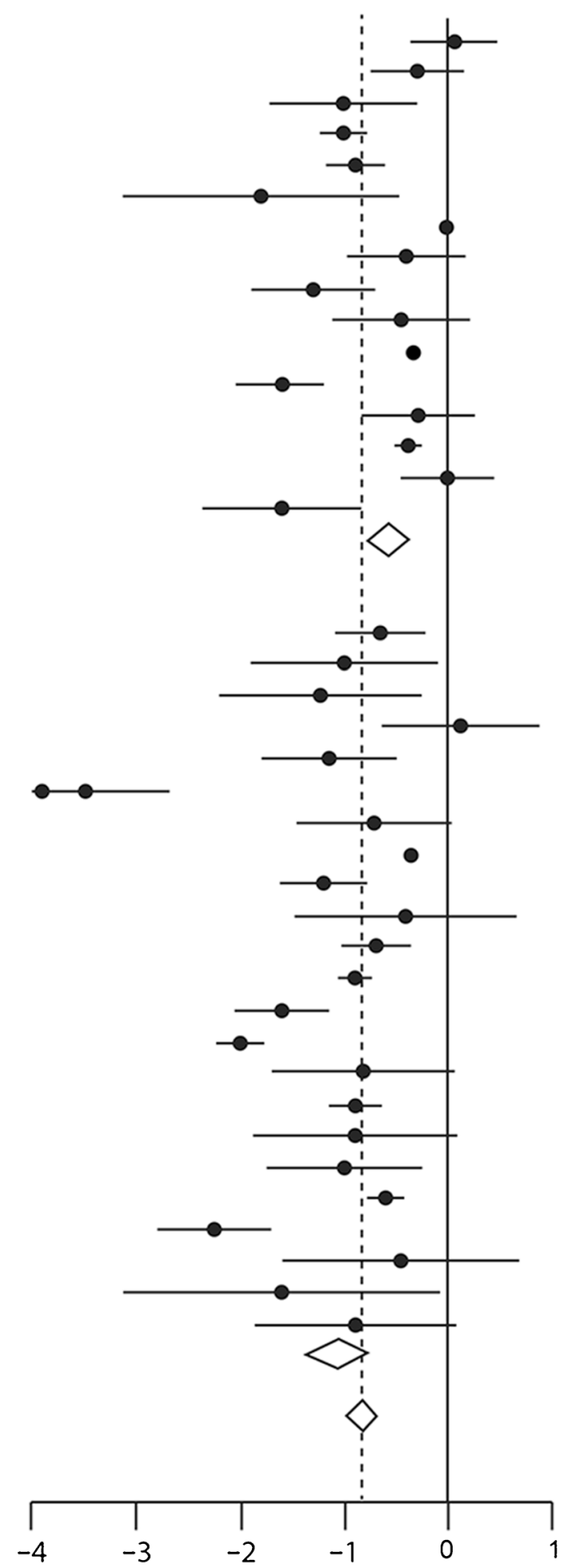

$0.06(-0.36,0.48)$

$-0.30(-0.76,0.16)$

$-1.01(-1.72,-0.30)$

$-1.00(-1.22,-0.78)$

$-0.90(-1.18,-0.62)$

$-1.80(-3.13,-0.47)$

$-0.03(-0.06,0.00)$

$-0.40(-0.97,0.17)$

$-1.30(-1.91,-0.69)$

$-0.45(-1.11,0.21)$

$-0.35(-0.38,-0.32)$

$-1.60(-2.02,-1.18)$

$-0.30(-0.85,0.25)$

$-0.38(-0.52,-0.24)$

$0.00(-0.44,0.44)$

$-1.60(-2.36,-0.84)$

$-0.59(-0.76,-0.42)$

$-0.66(-1.09,-0.23)$

$-1.00(-1.89,-0.11)$

$-1.23(-2.22,-0.24)$

$0.12(-0.65,0.89)$

$-1.14(-1.78,-0.50)$

$-3.50(-4.32,-2.68)$

$-0.71(-1.46,0.04)$

$-0.38(-0.39,-0.33)$

$-1.20(-1.61,-0.79)$

$-0.40(-1.46,0.66)$

$-0.70(-1.03,-0.37)$

$-0.90(-1.06,-0.74)$

$-1.60(-2.06,-1.14)$

$-2.00(-2.23,-1.77)$

$-0.82(-1.70,0.06)$

$-0.90(-1.17,-0.63)$

$-0.90(-1.87,0.07)$

$-1.00(-1.74,-0.26)$

$-0.60(-0.79,-0.41)$

$-2.26(-2.81,-1.71)$

$-0.46(-1.61,0.69)$

$-1.60(-3.13,-0.07)$

$-0.89(-1.86,0.08)$

$-1.07(-1.36,-0.78)$

$-84(-0.97,-0.71)$

Figure $2 \mathbf{H b A}_{1 \mathrm{c}}$ improvement: random-effects meta-analysis. Dark circles represent the $\mathbf{H b A}_{1 \mathrm{c}}$ change in individual studies; lines are $\mathbf{9 5 \%}$ confidence intervals. The diamond represents the pooled effect, and its width is the $95 \%$ confidence interval of the pooled estimate. Interventions were associated with a $0.84 \%$ reduction in $\mathrm{HbA}_{1 \mathrm{c}}$; this decrease was statistically significant but very heterogeneous across studies (95.9\% of heterogeneity was not explained by chance). $\mathrm{HbA}_{1 \mathrm{c}}=$ hemoglobin $\mathrm{A}_{1 \mathrm{c}}$; LEP = limited English proficiency; RCT = randomized controlled trial; $\mathrm{WMD}=$ weighted mean difference.

ascertained statistically because of the heterogeneity, which makes funnel plots unreliable. The certainty in the effectiveness of these interventions on hard endpoints (e.g., T2DM complications and microvascular or macrovascular 


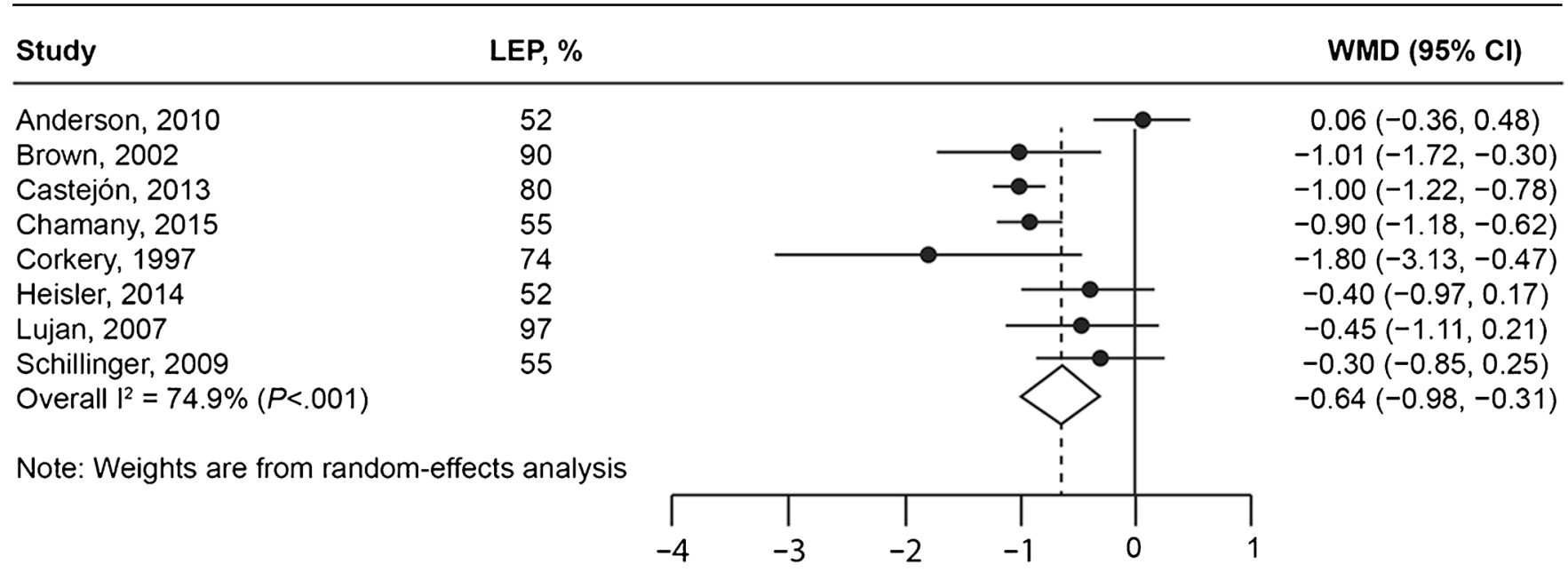

Figure 3 Sensitivity analysis of the effect on $\mathrm{HbA}_{1 \mathrm{c}}$ in studies with $\geq 50 \%$ of patients having limited English proficiency. $\mathrm{Hb} \mathrm{A}_{1 \mathrm{c}}=$ hemoglobin $A_{1 c} ; L E P=$ limited English proficiency; WMD = weighted mean difference.

disease) is low because the available evidence was indirect and focused on surrogate outcomes.

\section{DISCUSSION}

\section{Main Findings}

We conducted a systematic review and meta-analysis of the literature evaluating interventions for T2DM management among patients with LEP in North America. The interventions were associated with a heterogeneous but statistically significant reduction in $\mathrm{HbA}_{1 \mathrm{c}}(-0.84 \%)$. This degree of reduction in $\mathrm{HbA}_{1 \mathrm{c}}$ is clinically significant; a decrease of $0.9 \%$ is associated with a $25 \%$ reduction in microvascular complications and significant decreases in T2DM-related mortality and all-cause mortality. ${ }^{31}$ The overall effect is equivalent to or better than what would be expected with the addition of many pharmacotherapy agents used in the management of T2DM. Although the studies in this review had high heterogeneity, the metaanalysis showed a significant reduction in $\mathrm{HbA}_{1 \mathrm{c}}$ and further presented an opportunity to investigate the effectiveness of various elements of these complex interventions in the subgroup analysis. ${ }^{32}$ The majority of studies did not report attrition rates by LEP status. In studies with larger proportions of patients with LEP $(\geq 50 \%)$, overall attrition rates ranged from $12 \%$ to $33 \%$, with the exception of three outliers, two with a $5 \%$ rate and one with a $48 \%$ attrition rate. This is comparable to reports in the literature examining effectiveness of diabetes care management among non-LEP populations. ${ }^{33}$

Conclusions of this review are limited by the fact that no individual study included only patients with LEP. In 24 of the 54 studies, the exact proportion of patients with LEP was not reported, and we inferred percentages on the basis of intervention characteristics such as references to interpreter use and use of study materials in non-English languages. Furthermore, the results suggested that improvement in $\mathrm{HbA}_{1 \mathrm{c}}$ was more common in studies with a lower proportion of patients with LEP. This finding may be attributable to the fact that most of these interventions were not designed specifically for patients with LEP. Adaptation of health education and study materials for patients with LEP goes beyond translation to ensure appropriate cultural adaptation. $^{34-36}$

Most of the studies reported multicomponent interventions targeting several different T2DM self-management constructs, such as medication adherence, dietary management, physical activity, and self-monitored blood glucose. The cumulative success of these interventions highlights the importance of incorporating multiple interventions to address the complex behavior change necessary to effect a meaningful improvement in T2DM outcomes. Furthermore, because each intervention was crafted in the context of different patient populations and clinical resources, a reproducible, "one-size-fits-all" intervention package may not exist. Instead, practices should choose to implement aspects of different intervention components in their settings. Nevertheless, the heterogeneity of interventions limits the conclusions that can be drawn regarding the effectiveness of any specific components. These data are further limited by a paucity of process evaluations reported for the included studies, which facilitate a richer interpretation of outcomes by elucidating the factors that contributed to the success (or failure) of an intervention. ${ }^{37}$ Strong evidence suggests that the number of components of an intervention and the fidelity to intervention components directly impacts study outcomes, ${ }^{38}$ particularly for multimodal interventions such as those used in most of the studies included in this review. ${ }^{39}$

The subgroup analyses suggested greater $\mathrm{HbA}_{1 \mathrm{c}}$ reduction when interventions were delivered face-to-face through multiple sessions and with a $\mathrm{CHW}$ as the interventionist. Although some studies showed significant 
Table 1 Subgroup Analysis for the Effect on $\mathbf{H b A}_{1 c}$

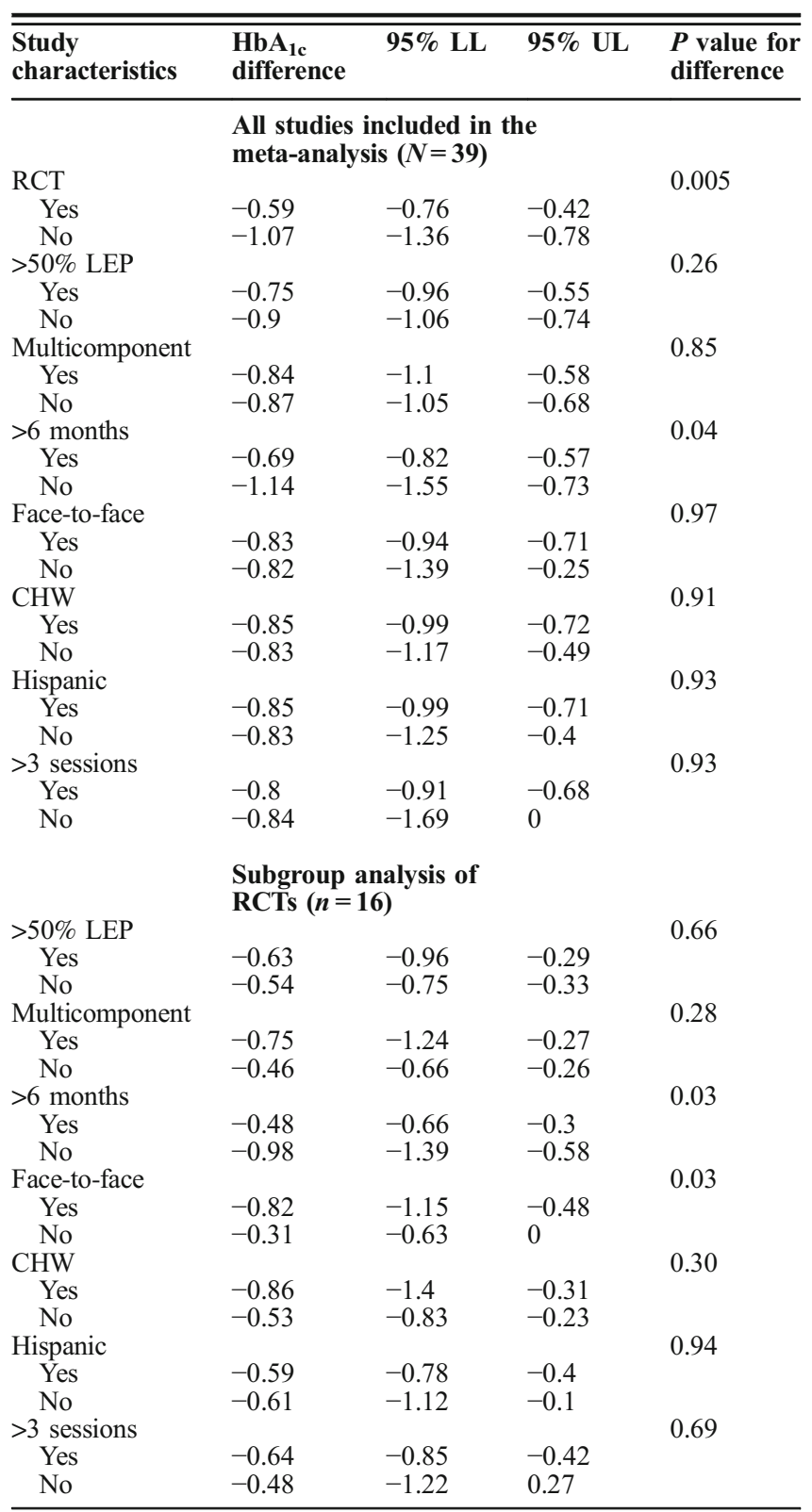

$C H W$, community health worker; HbA $A_{I c}$ hemoglobin $A_{I c} ; L E P$, limited English proficiency; LL, lower limit; RCT, randomized controlled trial; UL, upper limit

improvement in T2DM outcomes when a member (or members) of the clinical team served as the interventionist, CHWs and peer leaders appear to have a particularly important role in diabetes management for patients with LEP. ${ }^{40} \mathrm{CHW}$ are health outreach professionals who apply their unique understanding of the experience, language, and culture of the population they serve to carry out various roles that range from cultural mediation between individuals, communities, and health care teams, to providing direct services, education, and advocacy for individual or community needs. LEP is frequently associated with recent immigration, lower acculturation, lower health literacy, socioeconomic vulnerability, and difficulties in navigating the health care system. ${ }^{41}$ These barriers and the language discordance with the mainstream health care system increase difficulties for patients with LEP who are living with and managing T2DM. Unfortunately, these additional barriers are particularly challenging for health care teams as well, because they are beyond their scope of direct impact. CHWs and others from the communities where patients live are better positioned to help patients navigate through local resources; by partnering with such individuals, clinical practices may begin to overcome some of the sociodemographic barriers to T2DM management. ${ }^{42}$ Incorporation of CHWs into care teams can further extend the reach of these teams and may serve as an extension of care coordination for patients with T2DM. ${ }^{43-46}$

Only a few studies examined patient-reported outcomes or quality of life. This is consistent with the chronic disease literature more broadly, because it demonstrates a suboptimal focus on patient-related outcomes; however, such outcomes are increasingly recognized as an important supplement to physiological and biological measures of health status. ${ }^{47}$ A systematic examination is needed to identify factors that are important to patients who have a chronic disease that affects multiple facets of their lives; such factors should be incorporated into interventions to improve outcomes and reduce health disparities. This intentional design is particularly important for patients with LEP, who have worse patient-reported outcomes in some chronic diseases than do Englishproficient patients.

\section{Practical Implications}

The results of this review suggest that improvement in $\mathrm{HbA}_{1 \mathrm{c}}$ is more likely in studies with shorter interventions, administered face-to-face. Other factors associated with greater $\mathrm{HbA}_{1 \mathrm{c}}$ reduction were multicomponent interventions, with more than three sessions, delivered face-toface, and having a CHW as the interventionist. Complex behavior change is often necessary to achieve meaningful improvement in $\mathrm{T} 2 \mathrm{DM}$ outcomes, and consideration of patient context is critical. This implies that practices should adapt aspects of different intervention components to their settings to meet the specific linguistic and cultural needs of patients with LEP, guided by the ethnic and language composition of the patients that they serve. Given their unique capacity and placement in the community, CHWs can further address the complex challenges that affect patients with LEP, and may serve as an extension of clinical care teams. Because patients with LEP often constitute a small proportion of the total population served in clinical practices, interventions for chronic disease management are likely designed, by default, largely for patients who are proficient in English. Thus, clinics and practices should be purposeful in adapting these 
interventions to meet the specific linguistic and cultural needs of patients with LEP, whose composition may differ based on local population demographics. It is also important that future research specifically seek to answer important questions about patient outcomes and attrition on the basis of language proficiency.

\section{Limitations}

Although this is the first review that, to our knowledge, focuses on interventions for T2DM management among patients with LEP, we acknowledge several limitations. First, none of the studies included only patients with LEP, and in almost half of the studies, the percentage of patients with LEP could not be conclusively determined. This limitation is a direct result of the available studies and not a flaw in the review process, but it limits the conclusions that can be drawn on the effectiveness of the interventions. Second, heterogeneity in the design and characteristics of the interventions further limits the ability to draw conclusions, as well as to make recommendations. However, the results of this meta-analysis were robust, with sensitivity analysis showing consistent outcomes. Third, we observed significant heterogeneity in outcomes examined, with a limited number of studies reporting patient-related outcomes and quality of life. However, most studies measured $\mathrm{HbA}_{1 \mathrm{c}}$ levels, and most reported enough data to allow a meta-analysis. Fourth, few studies included patients from Africa or the Middle East, regions known to have an especially high prevalence of T2DM and poor T2DM outcomes. ${ }^{5,} 48$ Finally, even when proportions of patients with LEP were identified at baseline, outcomes were not adjusted for English proficiency status; thus, we could not determine exactly how interventions affected patients with LEP.

\section{CONCLUSION}

Multiple types of interventions are available for T2DM management among patients with LEP. Multicomponent interventions delivered face-to-face seem most effective for glycemic control. More research is needed to better understand other aspects of multicomponent interventions that are critical for improving important outcomes for patients with T2DM and LEP. Physician-specific educational tools must be developed to facilitate care of patients with T2DM and LEP.

Acknowledgments: This publication was made possible by funding from the Mayo Clinic Robert D. and Patricia E. Kern Center for the Science of Health Care Delivery.

Corresponding Author: Jane W. Njeru, MB, ChB; Division of Primary Care Internal Medicine, Mayo Clinic, Rochester, MN, USA (e-mail: njeru.jane@mayo.edu).

\section{Compliance with Ethical Standards:}

Conflict of Interest: The authors declare that they do not have a conflict of interest.

\section{REFERENCES}

1. Centers for Disease Control and Prevention. US Department of Health and Human Services. National diabetes fact sheet: national estimates and general information on diabetes and prediabetes in the United States, 2011 [Internet]. Atlanta (GA); 2011 [cited 8 Dec 2016]. Available from: http://www.cdc.gov/diabetes/pubs/pdf/ndfs_2011.pdf.

2. American Diabetes Association. Standards of medical care in diabetes: 2008. Diabetes Care. 200831 Suppl 1:S12-54.

3. Creatore MI, Moineddin R, Booth G, Manuel DH, DesMeules M, McDermott S, et al. Age- and sex-related prevalence of diabetes mellitus among immigrants to Ontario, Canada. CMAJ. 2010 182(8):781-9.

4. Lanting LC, Joung IM, Mackenbach JP, Lamberts SW, Bootsma AH. Ethnic differences in mortality, end-stage complications, and quality of care among diabetic patients: a review. Diabetes Care 2005 28(9):2280-8.

5. Wieland ML, Morrison TB, Cha SS, Rahman AS, Chaudhry R. Diabetes care among Somali immigrants and refugees. J Community Health 2012 37(3):680-4.

6. Heisler M, Faul JD, Hayward RA, Langa KM, Blaum C, Weir D. Mechanisms for racial and ethnic disparities in glycemic control in middle-aged and older Americans in the health and retirement study. Arch Intern Med 2007 167(17): 1853-60.

7. Li K, Wen $\mathbf{M}$. Racial and ethnic disparities in leisure-time physical activity in California: patterns and mechanisms. Race Soc Probl 2013 5(3): 147-156.

8. Schneiderman N, Llabre M, Cowie CC, Barnhart J, Carnethon M, Gallo LC, et al. Prevalence of diabetes among Hispanics/Latinos from diverse backgrounds: the Hispanic Community Health Study/Study of Latinos (HCHS/SOL). Diabetes Care 2014 37(8):2233-9.

9. Osborn CY, Cavanaugh K, Wallston KA, Kripalani S, Elasy TA, Rothman RL, et al. Health literacy explains racial disparities in diabetes medication adherence. J Health Commun 2011; 16 Suppl 3:268-78.

10. Rovner BW, Casten RJ, Harris LF. Sociocultural influences on diabetes self-management behaviors in older African Americans. Diabetes Spectr 2013 26(1):29-33.

11. Gaskin DJ, Thorpe RJ Jr, McGinty EE, Bower K, Rohde C, Young JH, et al. Disparities in diabetes: the nexus of race, poverty, and place. Am J Public Health 2014 104(11):2147-55.

12. Pandya C, Batalova J, McHugh M. Limited English proficient individuals in the United States: number, share, growth, and linguistic diversity [Internet]. Washington (DC): Migration Policy Institute; 2011 [cited 2016 Dec 8]. Available from: http://www.migrationinformation.org/integration/LEPdatabrief.pdf.

13. Agency for Healthcare Research and Quality. US Department of Health and Human Services. Medical expenditure panel survey: demographics and health care access and utilization of limited-English-proficient and English-proficient Hispanics [Internet]. Rockville (MD); 2008, Research Findings No. 28 [cited 8 Dec 2016]. Available from: https://meps.ahrq. gov/data_files/publications/rf28/rf28.pdf.

14. DuBard CA, Gizlice Z. Language spoken and differences in health status, access to care, and receipt of preventive services among US Hispanics. Am J Public Health 2008 98(11):2021-8.

15. Flores G, Tomany-Korman SC. The language spoken at home and disparities in medical and dental health, access to care, and use of services in US children. Pediatrics. 2008 121(6):e1703-14. Erratum in: Pediatrics. 2009 124(4): 1265.

16. Wilson E, Chen AH, Grumbach $\mathbf{K}$, Wang F, Fernandez A. Effects of limited English proficiency and physician language on health care comprehension. J Gen Intern Med 2005 20(9):800-6.

17. Murray MD, Tu W, Wu J, Morrow D, Smith F, Brater DC. Factors associated with exacerbation of heart failure include treatment adherence and health literacy skills. Clin Pharmacol Ther 2009 85(6):651-8.

18. Wisnivesky JP, Krauskopf $\mathbf{K}$, Wolf MS, Wilson EA, Sofianou A, Martynenko M, et al. The association between language proficiency and outcomes of elderly patients with asthma. Ann Allergy Asthma Immunol 2012 109(3):179-84. 
19. Bauer AM, Chen CN, Alegria M. English language proficiency and mental health service use among Latino and Asian Americans with mental disorders. Med Care 2010 48(12): 1097-104.

20. John-Baptiste A, Naglie G, Tomlinson G, Alibhai SM, Etchells E, Cheung A, et al. The effect of English language proficiency on length of stay and in-hospital mortality. J Gen Intern Med 2004 19(3):221-8.

21. Schenker Y, Karter AJ, Schillinger D, Warton EM, Adler NE, Moffet HH, et al. The impact of limited English proficiency and physician language concordance on reports of clinical interactions among patients with diabetes: the DISTANCE study. Patient Educ Couns 2010 81(2):222-8.

22. Choi S, Lee JA, Rush E. Ethnic and language disparities in diabetes care among California residents. Ethn Dis 2011 21(2):183-9.

23. Flores G. The impact of medical interpreter services on the quality of health care: a systematic review. Med Care Res Rev 2005 62(3):255-99.

24. Hacker K, Choi YS, Trebino L, Hicks L, Friedman E, Blanchfield B, et al. Exploring the impact of language services on utilization and clinical outcomes for diabetics. PLoS One 2012; 7(6):e38507.

25. Moher D, Liberati A, Tetzlaff J, Altman DG; PRISMA Group. Preferred reporting items for systematic reviews and meta-analyses: the PRISMA statement. PLoS Med. 2009 6(7):e1000097.

26. Higgins JPT, Altman DG, Sterne JAC. Assessing risk of bias in included studies. Chapter 8. In: Higgins JPT, Green S, (eds). Cochrane handbook for systematic reviews of interventions. Version 5.1.0; 2011.

27. Wells GA, Shea B, O'Connell D, Peterson J, Welch V, Losos M, et al. The Newcastle-Ottawa Scale (NOS) for assessing the quality of nonrandomised studies in meta-analyses [Internet]. Ottawa Hospital Research Institute [cited 8 Dec 2016]. Available from: http://www.ohri. ca/programs/clinical epidemiology/oxford.asp.

28. DerSimonian R, Laird N. Meta-analysis in clinical trials. Control Clin Trials 1986 7(3):177-88.

29. Higgins JP, Thompson SG, Deeks JJ, Altman DG. Measuring inconsistency in meta-analyses. BMJ 2003 327(7414):557-60.

30. Balshem H, Helfand M, Schunemann HJ, Oxman AD, Kunz R, Brozek J, et al. GRADE guidelines: 3. Rating the quality of evidence. J Clin Epidemiol 2011 64(4):401-6.

31. UK Prospective Diabetes Study (UKPDS) Group. Effect of intensive bloodglucose control with metformin on complications in overweight patients with type 2 diabetes (UKPDS 34). Lancet. 1998 352(9131):854-65. Erratum in: Lancet 1998 352(9139): 1558.

32. Murad MH, Almasri J, Alsawas M, Farah W. Grading the quality of evidence in complex interventions: a guide for evidence-based practitioners. Evid Based Med 2017 22(1):20-2.

33. Renders CM, Valk GD, Griffin SJ, Wagner EH, Van Eijk JT, Assendelft WJ. Interventions to improve the management of diabetes in primary care, outpatient, and community settings: a systematic review. Diabetes Care 2001 24(10):1821-33.

34. McElroy A, Jezewski MA. Cultural variation in the experience of health and illness. In: Albrecht GL, Fitzpatrick R, Scrimshaw SC, editors. The handbook of social studies in health and medicine. Thousand Oaks: Sage Publications Ltd; 2000. p. 191-209.
35. Tabak RG, Sinclair KA, Baumann AA, Racette SB, Sebert Kuhlmann A, Johnson-Jennings MD, et al. A review of diabetes prevention program translations: use of cultural adaptation and implementation research. Transl Behav Med 2015 5(4):401-14.

36. Carter JS, Gilliland SS, Perez GE, Levin S, Broussard BA, Valdez L, et al. Native American Diabetes Project: designing culturally relevant education materials. Diabetes Educ 1997 23(2):133-4, 139.

37. Rychetnik L, Frommer M, Hawe P, Shiell A. Criteria for evaluating evidence on public health interventions. J Epidemiol Community Health 2002 56(2):119-27.

38. Durlak JA, DuPre EP. Implementation matters: a review of research on the influence of implementation on program outcomes and the factors affecting implementation. Am J Community Psychol 2008 41(3-4):32750

39. Oakley A, Strange V, Bonell C, Allen E, Stephenson J; RIPPLE Study Team. Process evaluation in randomised controlled trials of complex interventions. BMJ 2006 332(7538):413-6.

40. Kane EP, Collinsworth AW, Schmidt KL, Brown RM, Snead CA, Barnes SA, et al. Improving diabetes care and outcomes with community health workers. Fam Pract 2016 33(5):523-8.

41. Zong J, Batalova, J. The limited English proficient population in the United States [Internet]. Washington (DC): Migration Policy Institute; 2015 [cited 2016 Dec 8]. Available from: http://www.migrationpolicy.org/ article/limited-english-proficient-population-united-states.

42. Walker RJ, Strom Williams J, Egede LE. Influence of race, ethnicity and social determinants of health on diabetes outcomes. Am J Med Sci 2016 351(4):366-73.

43. Cosgrove S, Moore-Monroy M, Jenkins C, Castillo SR, Williams C, Parris E, et al. Community health workers as an integral strategy in the REACH US program to eliminate health inequities. Health Promot Pract 2014 15(6):795-802

44. Cherrington A, Ayala GX, Amick H, Allison J, Corbie-Smith G, Scarinci I. Implementing the community health worker model within diabetes management: challenges and lessons learned from programs across the United States. Diabetes Educ 2008 34(5):82433

45. Spiro A, Oo SA, Marable D, Collins JP. A unique model of the community health worker: the MGH Chelsea Community Health Improvement team. Fam Community Health 2012 35(2):147-60.

46. Collinsworth A, Vulimiri M, Snead C, Walton J. Community health workers in primary care practice: redesigning health care delivery systems to extend and improve diabetes care in underserved populations. Health Promot Pract 2014 15(2 Suppl):51S-61S.

47. Gabriel SE, Normand SL. Getting the methods right: the foundation of patient-centered outcomes research. N Engl J Med 2012 367(9):787-90.

48. Njeru JW, Tan EM, St Sauver J, Jacobson DJ, Agunwamba AA, Wilson PM, et al. High rates of diabetes mellitus, pre-diabetes and obesity among Somali immigrants and refugees in Minnesota: a retrospective chart review. J Immigr Minor Health 2016 18(6): 1343-9 